Conclusion: In our cohort the majority of PsA pts had mild PsO preceded PsA on average of $9.2 \mathrm{yrs}$. Bone erosion was found in $30 \%$ of PsA pts which associated with $\mathrm{PsO}$ duration, skin and nail disease severity as well as with PsA activity. Early diagnosis and therapeutic intervention within a "window of opportunity" are very important for improving outcomes and prevent structural damage in PsA. REFERENCES:

[1] Tillett W, et al. Interval between onset of psoriasis and psoriatic arthritis comparing the UK Clinical Practice Research Datalink with a hospital-based cohort. Rheumatol. 2017; 56, 2109-2113

[2] Scher JU, et al. Preventing psoriatic arthritis: focusing on patients with psoriasis at increased risk of transition. Nat Rev Rheumatol. 2019;15(3):153-166. doi: 10.1038/s41584-019-0175-0. PMID: 30742092.

Disclosure of Interests: None declared.

DOI: 10.1136/annrheumdis-2021-eular.2796

\section{AB0577 METABOLIC SYNDROME IN PSORIATIC ARTHRITIS SEEMS ASSOCIATED TO INFLAMMATORY STATUS OF THE DISEASE}

C. L. Iñíguez ${ }^{1}$, J. A. Mosquera Martínez², L. Fernández-Dominguez ${ }^{3}$, F. Maceiras-Pan ${ }^{4}$, C. García-Porrúa ${ }^{1}$, J. L. Guerra-Vázquez ${ }^{5}$, V. Quevedo Vila $^{6}$, M. Caeiro-Aguado ${ }^{7}$, J. Lorenzo-Alvarez ${ }^{8}$, J. A. Pinto Tasende ${ }^{7,9}$ on behalf of GIGAPs. ${ }^{1}$ Hospital Lucus Augusti, Rheumatology, Lugo, Spain; ${ }^{2}$ Hospital Montecelo (Pontevedra), Rheumatology, Pontevedra, Spain; ${ }^{3}$ Complexo Hospitalario Universitario de Ourense, Rheumatology, Ourense, Spain; ${ }^{4}$ Hospital do Meixoeiro, Rheumatology, Vigo, Spain; ${ }^{5}$ Hospital Arquitecto Marcide, Rheumatology, Ferrol, Spain; ${ }^{6}$ Hospital Comarcal de Monforte de Lemos, Rheumatology, Monforte de Lemos, Spain; ${ }^{7}$ Hospital Universitario da Coruña, Rheumatology, La Coruña, Spain; ${ }^{8}$ Hospital Universitario da Coruña, Internal Medicine, La Coruña, Spain; ${ }^{9}$ INIBIC, Rheumatology, A Coruña, Spain

Background: Evidence suggests that inflammation plays a causal role in the development of Metabolic Syndrome (MetS) and elevated peripheral levels of proinflammatory mediators, such as C-reactive protein (CRP) confering cardiovascular and metabolic risk.

Objectives: The objective of this study was to evaluate Metabolic Syndrome among patients with PsA naïve to biologics and to evaluate its association with inflammatory activity status previous start them.

Methods: We performed a retrospective cross-sectional study of a cohort of patients $\geq 18$ years of age, all patients with a diagnosis of PSA (CASPAR criteria), included in the Sueiro Cohort. Patients were managed according to a standard protocol adopted at the Rheumatology outpatient clinic of six hospitals and they were patients on follow-up for at least 6 months. Collected variables included age and gender, blood biochemistry, blood count, erythrocyte sedimentation rate (ESR), C-reactive protein (CRP), HLA-B27 and rheumatoid factor, glucocorticoid, NSAID and conventional or biologic DMARD, smoking habit, hyperuricemia, type 2 diabetes mellitus, obesity (BMI kg/m2). MetS was defined by a combination of abdominal obesity, impaired fasting glucose, atherogenic dyslipidemia, and elevated blood pressure. Status of disease activity was supported by tender and swollen joint counts (TJC68 and SJC66) from patients who were going to start treatment with biologics and Patient-reported outcome measure were collected with Patient Global Assessment $(0-10 \mathrm{~cm})$ and Patient Pain Assessment $(0-10 \mathrm{~cm})$. Remission and low disease activity status was obtained by DAPSA score and its defined cut-off. Tests were two-tailed with a significance level of 5\%. Data were analyzed using SPSS V21.0 statistical software (IBM Corp. NY, USA).

Results: A total of 416 were included in the study: 222 PsA patients treated with csDMARD kept in remission or low disease activity and 194 patients needed to be treated with bDMARD. The mean age of the patients was 53.0 years (SD: 11.8). Two hundred and twenty-eight were males (56.2\%).

Fifty-six patients had MetS (13.8\%) and it was more frequent in patients who were in clinical activity and they needed biologics (17.9\% vs $10.4 \%, \mathrm{p} 0.028$ ) with mean (SD) of DAPSA of 16.7 (11.1). Patients starting treatment with biologics and with MetS had more proportion of patients older than 50 years $(24.2 \%$ vs $8.3 \%$, p 0.006) and CRP $>5 \mathrm{mgr} / \mathrm{L}(71.4 \%$ vs $52.6 \%$, p 0.042$)$ and DAPSA had higher values than in patients without MetS (18.6 vs 16.2) but there were not significant statistical differences. Binary regression analysis showed increased risk of MetS for age $>50$ years old (OR 3.287 [95\% $\mathrm{Cl}$ : 1.258-8.591], p 0.015) and CRP > 5 mgr/L (OR 2.684 [95\%Cl: 1.141-6.313], p 0.024) but not for gender (OR 2.136 [95\%Cl: 0.932-4.893], p 0.073) neither for DAPSA>14 (OR 1.539 [95\%Cl: 0.695-3.409], p 0.288)

Conclusion: Patients with PsA active despite csDMARD had more prevalence of MetS and this was associated with those patients over 50 years old and CRP higher than normal values. DAPSA was higher in patients with MetS but without reaching significant statistical difference.

REFERENCES:

[1] Lee $\mathrm{YH}$, Pratley RE. The evolving role of inflammation in obesity and the metabolic syndrome. Current Diabetes Reports. 2005;5:70-75
Acknowledgements: I have acknowledgements to SOGARE

Disclosure of Interests: None declared.

DOI: 10.1136/annrheumdis-2021-eular.2947

\section{AB0578 SUBCLINICAL ENTHESITIS IN PSORIASIS PATIENTS AS PREDICTOR OF ARTHRITIS (EPESCA STUDY): PRELIMINARY RESULTS}

E. Di Donato ${ }^{1}$, A. Becciolini ${ }^{1}$, S. DI Nuzzo ${ }^{2}$, N. Chernyschova ${ }^{3}$, C. Commisso ${ }^{4}$ S. Lamorte ${ }^{4}$, M. Brusasco ${ }^{2}$, E. Pierobon ${ }^{2}$, D. Santilli' ${ }^{1}$, G. Lucchini ${ }^{1}$, M. Riva ${ }^{1}$, N. Sverzellati ${ }^{4}$, F. Mozzani ${ }^{1}$, A. Ariani ${ }^{1}{ }^{1}$ Azienda Ospedaliero-Universitaria di Parma, Internal Medicine and Rheumatology Unit, Parma, Italy; ${ }^{2}$ University of Parma, Department of Medicine and Surgery, Parma, Italy; ${ }^{3}$ Azienda Ospedaliero-Universitaria, Section of Radiology, Parma, Italy; ${ }^{4}$ University of Parma, Department of Radiological Sciences, Parma, Italy

Background: Enthesitis is one of the typical pathological signs of spondyloarthritis such as psoriatic arthritis ( $\mathrm{PsA}$ ) and it seems to be the Primum movens of the disease. Clinical assessment of enthesitis showed to be less sensitive, compared to ultrasound (US) evaluation, in identifying enthesitis in patients with PsA [1].

OMERACT defined US enthesitis as: "hypoechoic and/or thickened insertion of the tendon close to the bone (within $2 \mathrm{~mm}$ from the bony cortex), which exhibits Doppler signal if active and that may show erosions, enthesophytes/calcifications as a sign of structural damage" [3]

The reported prevalence of subclinical enthesitis in psoriasis ( $\mathrm{PsO}$ ) patients in different countries ranges between $7 \%$ and $20 \%$ [2].

Objectives: The main objective of this study was to estimate, by US evaluation, the prevalence of subclinical enthesitis in $\mathrm{PsO}$ patients without any clinical signs of enthesitis. Secondary objectives were to analyze differences, in terms of age, sex, BMI, PsO onset and diagnosis, among patients with enthesitis (active or not active), enthesopathy and without any alteration of enthesis.

According to the OMERACT definition of ultrasound enthesitis, patients were divided into 4 groups: patients with active enthesitis $(A E)$ defined as the presence of power-Doppler signal in a hypoechoic and/or thickened insertion of the tendon close to the bone; patient with enthesitis (En) defined as hypoechoic and/or thickened insertion of the tendon close to the bone without PD signal; patients with enthesopathy (Ep) defined as the presence of structural damage (erosions, enthesophytes/calcifications); patients without any alteration of enthesis (WE).

Methods: Patients with at least 18 years and a diagnosis of $\mathrm{PsO}$ made by a Dermatologist were included. Exclusion criteria were the presence of clinical symptoms or signs of articular or entheseal involvement, diagnosis of arthritis and therapy with bDMARDs or tsDMARDs.

All patients underwent US examination on grey scale and Power Doppler (PD) ultrasonography of 6 sites (Achilles, quadriceps, distal and proximal patellar plantar fascia and triceps enthesis) bilaterally. Ultrasound was performed by an experienced sonographer, using a Logiq P9 equipped with 6-12 $\mathrm{MHz}$ broad band linear transducer. Data were reported as frequencies and median with interquartile range. To check differences among these four groups, we used chi-square test or Kruskall-Wallis test. P-value $\leq 0.05$ is considered statistically significant.

Results: We enrolled 124 consecutive psoriasis patients (47 [37.9\%] female) with median age 57.7 (45.3-66.5) years, median disease duration 20.4 (10.1 30.8) years, median BMI 27 [24-29]. Patients with AE, En, Ep were $20.2 \%$ (25/124), 49.2\% (61/124) and $18.5 \%(23 / 124)$, respectively.

Patients WE $(12.1 \%, 15 / 124)$ showed significantly lower BMI, younger age and shorter diagnostic delay compared to the other patients.

All signs of enthesopathy/enthesitis were more frequently observed at Achilles (33.1\%, 41/124), triceps $(23.4 \%, 29 / 124)$, quadriceps $(20.2 \%, 25 / 124)$, distal patellar $(18.5 \%, 23 / 124)$ and proximal patellar $(4.8 \%, 6 / 124)$ enthesis.

Conclusion: Subclinical enthesitis is quite common in $\mathrm{PsO}$ patients, and about $20 \%$ showed active enthesitis. The enthesopathy seems to be more frequent in patients with increased delay in $\mathrm{PsO}$ diagnosis, older age and higher BMI.

\section{REFERENCES:}

[1] Bandinelli F. et al. Ultrasound detects occult entheseal involvement in early psoriatic arthritis independently of clinical features and psoriasis severity. Clin Exp Rheumatology. Mar-Apr 2013;31(2):219-24.

[2] Zuliani F. et al. Ultrasonographic detection of subclinical enthesitis and synovitis: a possible stratification of psoriatic patients without clinical musculoskeletal involvement. Clin Exp Rheumatol Jul-Aug 2019;37(4):593-599.

[3] Balint P. V. et al. Reliability of a consensus-based ultrasound definition and scoring for enthesitis in spondyloarthritis and psoriatic arthritis: an OMERACT US initiative. Ann Rheum Dis 2018 Dec;77(12):1730-1735.

Disclosure of Interests: None declared.

DOI: 10.1136/annrheumdis-2021-eular.3149 